\title{
Caractéristiques agronomiques et physico-chimi- ques de lignées de tomate isogéniques, sauf pour le gène pat-2 de parthénocarpie, dans trois types variétaux, en culture de printemps, sous serre plastique très peu chauffée $\left({ }^{1}\right)$
} $\operatorname{NICOLAS~}(*)$

I.N.R.A., Station d'Amélioration des Plantes Maraîchères

(*) Laboratoire de Méthodes Physiques d'Etude (LAMPE), Centre de Recherches d'Avignon, F84140 Montfavet 3 types variétaux de tomate et les versions parthénocarpiques, isogéniques, sauf pour le gène pat-2 de parthénocarpie, de ces types variétaux, en culture au printemps sous serre plastique très peu chauffée.

Les variétés répondent de manière différente à l'introduction du gène pat-2. Ainsi, la version parthénocarpique de "Apédice" accuse un manque de vigueur important. Le rendement total des lignées parthénocarpiques est inférieur à celui des lignées normales dans le cas de "Apédice » et «Monalbo », ceci n'est pas dû à une diminution du nombre de fruits (le nombre de fruits noués est égal sinon supérieur chez les lignées parthénocarpiques), mais à une diminution du poids du fruit. D'une façon générale, la précocité est améliorée chez le matériel parthénocarpique.

Les caractéristiques physico-chimiques des fruits ont pu être comparées entre fruits sans graines des versions parthénocarpiques et fruits avec graines des témoins normaux ; la vibration des inflorescences n’a entraîné que très peu de fruits avec graines chez les lignées parthénocarpiques de "Monalbo » et "Porphyre », de telle sorte qu'il n’a pas été possible de comparer les fruits avec ou sans graincs issus de la même lignée parthénocarpique. Chez. "Apédice" parthénocarpique, les fruits avec graines sont apparus aux alentours de la même date, que les inflorescences aient été vibrées ou non; chez ce type variétal, les caractéristiques physiques des fruits avec et sans graines de la lignée parthénocarpique ont pu être comparés.

Les fruits des versions parthénocarpiques sont sensiblement moins fermes que les témoins. Chez "Apédice », les fruits sans graines de la lignée parthénocarpique sont moins lourds et moins fermes que les fruits avec graines de la même lignée. Chez les lignées parthénocarpiques des 3 types variétaux, la chair des fruits est plus rouge que chez les fruits des témoins correspondants : le gel à l"intéricur des loges est plus vert chez. "Monalbo » et "Porphyre " parthénocarpiques, plus rouge chez Apédice parthénocarpique.

Au niveau des caractéristiques chimiques des fruits, les lignées parthénocarpiques se distinguent, par rapport aux lignées témoins, par:

- une acidité titrable, un rapport cilrique/malique, une conductivité moins élevés en général,

une acidité citrique moins élevéc dans tous les cas testés.

- une teneur en matière sèche identique ou inféricure.

- des teneurs en lycopène et en carotène plus élevées chez certains types variétaux.

- une teneur en vitamine $C$ peut-厃̂̀tre inférieure.

Aucune tendance nette ne se dégage pour les autres critères analysés, à savoir la teneur en acide malique, le $\mathrm{pH}$. l'indice de réfraction, les sucres réducteurs.

Ces résultats, qui demanderaient à ĉtre précisés dans d'autres conditions de culture, et avec un matériel végétal diversifié (en particulier des types variétaux destinćs à l'industrie), permettent d'ores et déjà d'attirer l'attention du sélectionneur sur les caractéristiques modifiées par le gène pat-2, de façon à tirer profit des avantages de l'aptitude à la parthénocarpie naturelle (mise à fruits et précocité améliorées à basses températures), tout en minimisant ou en éliminant, par le choix de géniteurs appropriés, les caractéristiques moins favorables qui lui seraient associées.

Mots clés additionnels : Lycopersicon csculcntum, parthénocarpie naturelle. 
Agronomic and physicochemical characteristics of tomato lines isogenic except for the pat-2 gene for parthenocarpy, in three varietal types, in spring cultivation under a plastic house with very little heating.

This study was concerned with the agronomic performances and the physicochemical characteristics of fruits of 3 tomato varietal types, and the parthenocarpic lines, isogenic except for the pat-2 gene for parthenocarpy, of these varietal types, in spring cultivation under a plastic house, with very little heating. The varieties reacted in a different manner to the introduction of the pat-2 gene. For example, the parthenocarpic line of "Apédice" was much less vigorous than the normal line. The total yield of the parthenocarpic lines was less than that of the normal lines in the case of "Apédice" and "Monalbo". This was not due to a reduction in fruit number (the number of fruit set by the parthenocarpic lines was equal or higher), but to a reduction in fruit weight. As a general rule, earliness was improved in parthenocarpic material. It was possible to compare the physicochemical characteristics of seedless fruits from the parthenocarpic lines and seeded fruits from the control lines. However, after vibration of the inflorescences, there were only very few seeded fruits in the parthenocarpic lines of "Monalbo" and "Porphyre", so it was not possible to compare seedless and seeded fruits from the same parthenocarpic lines. In the parthenocarpic line of "Apédice", seeded fruits appeared around the same date, whether the inflorescences were vibrated or not: in this varietal type, it was possible to compare the physical characteristics of seeded and scedless fruits from the parthenocarpic line. The fruits from the parthenocarpic versions were appreciably less firm than the controls. In "Apedice", the seedless fruits from the parthenocarpic line were less heavy and less firm than the seeded fruits from the same line. In the parthenocarpic lines in the 3 varietal types, the red colour of the fruit flesh was more intense than the colour of the fruits from the corresponding controls; the gel inside the locules was more green in the parthenocarpic lines of "Monalbo" and Porphyre, more red in "Apédice". As regards the chemical properties of the fruits, the parthenocarpic lines were characterized by : titratable acidity, citric/malic ratio, conductivity lower in general; citric acidity lower in all the cases tested; dry matter contents identical or lower ; lycopene and carotene contents higher in some varietal Lypes; vitamin C contents possibly lower. No clear tendency was observed for the other characteristics analyzed: malic acid content, $\mathrm{pH}$, refractive index, reducing sugars. These results, which call for additional experimentation in different culture conditions with diversified plant material (in particular with varietal types for processing), allow here and now to draw the attention of the breeder to the characteristics modified by the pat- 2 gene, in order to make use of the advantages of natural parthenocarpy (fruit setting and earliness improved at low temperatures), and to minimize or to eliminate, by the choice of appropriate plant material, the less favorable characteristics that could be associated with it.

Additional key words : Lycopersicon esculentum, natural parthenocarpy.

\section{INTRODUCTION}

Différentes origines d'aptitude à la parthénocarpie naturelle sont actuellement disponibles chez la tomate (Philouze, 1983, 1985). Celle qui semble la plus directement utilisable vient de la variété russe "Severianin ». Elle est due au gène récessif pat-2 (parthenocarpic-2). Une bonne connaissance des incidences de ce gène sur les caractéristiques agronomiques et physico-chimiques des fruits est nécessaire, afin de permettre aux sélectionneurs d'entreprendre de manière plus efficace la création d'un matériel parthénocarpique performant.

Dans une étude préliminaire (non publiée), nous avons comparé à l'intérieur de la variété "Severianin ", en culture de plein champ, quelques caractéristiques physico-chimiques de fruits sans graines (obtenus sur des plantes dont les fleurs ont été castrées mais non pollinisées) et de fruits avec graines (obtenus sur des plantes dont les fleurs ont été castrées et pollinisées par du pollen de «Severianin»). Les quelques mesures effectuées ne nous ont pas permis de constater de différences entre les 2 types de fruits, pour la fermeté de la chair, le $\mathrm{pH}$ et l'indice de réfraction du jus, l'acidité titrable, les teneurs en sucres totaux et réducteurs.

CASAS-DIAZ et al. (1987) ont travaillé avec la lignée L-157 D, homozygote pour le gène pat-2, et en ségrégation pour la stérilité mâle : les plantes mâle-stériles ne donnent que des fruits sans graines, les plantes mâlefertiles un mélange de fruits avec et sans graines. Le pourcentage de sucres solubles des fruits sans graines récoltés sur plantes mâle-stériles est significativement plus élevé que celui des fruits avec peu de graines récoltés sur plantes mâle-fertiles, lui-même plus élevé (le plus souvent de façon significative) que les fruits avec graines nombreuses récoltés sur les mêmes plantes, et ceci pour 3 dates de plantation. Les fruits avec peu ou pas de graines ont en général un $\mathrm{pH}$ plus élevé que les fruits avec graines nombreuses.

Les résultats reportés ci-dessus portent sur des fruits avec ou sans graines à l'intérieur d'une même lignée parthénocarpique. En fait, il serait intéressant de comparer, à l'intérieur d'un même type variétal, une version normale et une version parthénocarpique et, à l'intérieur de cette dernière, des fruits avec ou sans graines. Dans ce but, nous avons créé un matériel isogénique, sauf pour le gène pat-2, dans 3 types variétaux : «Apédice», «Monalbo » et "Porphyre». Les résultats agronomiques d'un essai conduit en conditions non limitantes (sous serre verre chauffée avec plantation du 31 janvier) ont été publiés (Philouze, 1984). Dans cette étude, nous nous proposons maintenant d'évaluer les performances agronomiques de ce matériel en conditions limitantes de températures (culture au printemps sous serre plastique très peu chauffée). C'est en effet dans ces conditions difficiles que le matériel parthénocarpique peut présenter de l'intérêt par rapport au matériel normal, grâce à une mise à fruit précoce. Parallèlement nous avons effectué une étude exhaustive des caractéristiques physico-chimiques des fruits ainsi obtenus. 


\section{MATÉRIEL ET MÉTHODES}

\section{A. Matériel végétal}

Le gène pat-2 venant de "Severianin » a été introduit dans 3 lignées, représentant 3 types variétaux aux caractéristiques agronomiques bien différentes, sélectionnées à la Station d'Amélioration des Plantes Maraîchères de l'I.N.R.A.-Avignon: "Apédice», "Monalbo» et "Porphyre», lignées maintenant anciennes utilisées dans les programmes de sélection pour le marché de frais. La réalisation de l'hybride F1 entre "Severianin » et chacune des 3 lignées a été suivie de 5 rétrocroisements, puis 2 autofécondations ont permis de fixer le matériel. A ce niveau, on a retenu une lignée parthénocarpique pat-2/pat-2, ainsi qu'une lignée sœur non parthénocarpique $p a t-2^{+} / p^{2} t-2^{1}(\mathrm{ou}+/+)$, issue $\mathrm{du}$ même programme de rétrocroisements. Ce matériel est comparé à la lignée utilisée comme parent récurrent. La comparaison de la lignée parthénocarpique aux 2 lignées non parthénocarpiques doit permettre, dans le cas où des différences seraient observées, de faire la part entre ce qui est dû au caractère parthénocarpique (comparaison entre la lignée parthénocarpique et sa lignée sœur non parthénocarpique), et ce qui proviendrait d'un nombre insuffisant de rétrocroisements (comparaison entre la lignée non parthénocarpique ayant subi les 5 rétrocroisements, et le parent récurrent).

\section{B. Techniques culturales}

Le semis a été effectué en serre chauffée le 27 janvier, le repiquage le 14 février, la plantation le 15 mars sous serre plastique très peu chauffée. Les températures minima se sont situées entre 7 et $10{ }^{\circ} \mathrm{C}$ jusqu’à la fin avril, c'est-à-dire pendant l'essentiel de la période de floraison. Les plantes étaient tuteurées, conduites sur une tige taillée à 6 bouquets.

Les 3 types variétaux "Apédice», "Monalbo » et "Porphyre » ont été cultivés dans 3 essais séparés, sous forme de 3 carrés latins $4 \times 4$, soit 4 répétitions de 4 lots représentant les combinaisons suivantes: (1) lignée parthénocarpique, sans vibration des inflorescences, (2) lignée parthénocarpique, avec vibration des inflorescences, (3) lignée normale sœur de la lignée parthénocarpique, avec vibration des inflorescences, (4) lignée normale utilisée comme parent récurrent, avec vibration des inflorescences. Chaque parcelle élémentaire comportait 4 plantes, soit 64 plantes par type variétal et 192 plantes au total.

Les inflorescences des lots (2), (3) et (4) sont vibrées avec un vibreur électrique 2 à 3 fois par semaine, quand elles comportent au moins une fleur épanouie. $\mathrm{Ce}$ traitement qui favorise la chute du pollen, donc la fécondation et la nouaison, est nécessaire pour une production correcte de fruits chez les lignées non parthénocarpiques dans les conditions difficiles de l'essai ; il est utilisé dans la pratique. La vibration des inflorescences de la lignée parthénocarpique devait favoriser la production de fruits avec graines et permettre les comparaisons suivantes :

(1) fruits sans graines et fruits avec graines de la lignée parthénocarpique,
(2) fruits sans graines de la lignée parthénocarpique et fruits avec graines des lignées normales,

(3) fruits avec graines de la lignée parthénocarpique et fruits avec graines des lignées normales,

(4) enfin, fruits avec graines des 2 lignées normales témoins.

Nous verrons par la suite que la lignée parthénocarpique vibrée n'a donné que très peu de fruits avec graines chez «Monalbo » et "Porphyre », et cela tout au long de la récolte; les basses températures au moment de la floraison ont favorisé le développement parthénocarpique précoce du fruit. Chez « Apédice », la grande majorité des fruits de la version parthénocarpique ne contient pas de graines jusqu'aux environs du 17 juin : à partir de cette date, la majorité des fruits contient de nombreuses graines. Par rapport aux 2 autres types variétaux, «Apédice» se caractérise par sa richesse en pollen de bonne qualité, qui a ainsi assuré avec succès la fécondation dès que les températures minima ont dépassé $10^{\circ} \mathrm{C}$.

\section{Etude agronomique}

\section{Vigueur et hauteur des plantes}

Des observations ont été effectuées sur la vigueur des plantes. La hauteur des plantes a été mesurée, du niveau du sol au point d'insertion du $6^{\text {C }}$ bouquet, chez «Apédice » et "Monalbo"; les moyennes ont été comparées au moyen du test $t$ de Student. Dans le cas de « Porphyre ", dont le type de croissance est déterminé, de telles mesures n'ont pas été effectuées; cependant on a pu noter que les plantes de la version parthénocarpique avaient tendance à être moins végétatives que les témoins.

\section{Rendements et facteurs du rendement}

Tous les fruits mûrs ont été récoltés entre le 18 mai et le 6 juillet pour les analyses physico-chimiques. Afin que les résultats agronomiques soient complets, des récoltes supplémentaires ont été effectuées en mélangeant les fruits des 4 plantes de la même parcelle, le 19 juillet pour les 3 types variétaux, et le 30 juillet pour "Monalbo". L'analyse statistique a été faite en carré latin, et a porté sur les récoltes cumulées regroupées par semaine, sur le nombre de fruits récoltés et sur le poids moyen des fruits.

\section{Etude physico-chimique des fruits récoltés}

La méthodologie utilisée est celle décrite par DUPRAT et al. (1987).

\section{Mesures physiques}

Les fruits destinés aux analyses physiques et chimiques sont récoltés au stade mûr (orangé-rouge à rouge). La récolte est limitée à 5 bouquets par plante et à 10 fruits par bouquet; les fruits excédentaires sont prélevés et pesés pour être intégrés aux résultats agronomiques. Chaque tomate est placée dans un sac plas- 
tique numéroté. Au total 4696 fruits ont éte récoltés, répartis en 1953 fruits de "Apédice», 1608 fruits de «Monalbo », 1135 fruits de "Porphyre ». Pour chaque fruit, on repère les numéros de la parcelle, de la plante et du bouquet dont il est issu.

\section{Protocole des mesures physiques des fruits}

Neuf mesures physiques sont effectuées sur chacun des 4696 fruits récoltés. Les 5 premières mesures sont réalisées sur un même poste de travail, décrit par ailleurs (Nicolas et al., (1986). Le poids en g (intégré dans les résultats agronomiques) et la densité par rapport à l'eau sont obtenus par pesée. La compressibilité de la peau, les fermetés de la peau et de la chair sont mesurées au moyen d'un pénétromètre équipé d'un embout cylindrique comportant une première section de $2 \mathrm{~mm}$ de diamètre, longue de $6 \mathrm{~mm}$, suivie d'une seconde de $5 \mathrm{~mm}$ de diamètre. En fonction de la pénétration de l'embout, la force de résistance du fruit (exprimée en g) passe par 2 maxima, le premier est fonction de la fermeté de la peau, le second de celle de la chair. La compressibilité de la peau, exprimée en pourcent, correspond à la distance d'enfoncement de la pointe depuis son contact avec le fruit jusqu'à l'éclatement de la peau, rapportée au diamètre équatorial du fruit.

Après découpe équatoriale du fruit, on évalue 4 caractéristiques internes:

- l'importance des creux des fruits, selon 4 classes: de 1 (fruit plein) à 4 (fruit très creux);

- la couleur du gel, notée selon 4 classes: 1 : vertjaune, $2:$ orangé-rouge, $3:$ rouge, $4:$ rouge foncé ;

- la couleur de la chair, selon 3 classes : 1 : orangérouge, 2 : rouge, 3 : rouge foncé ;

- le nombre de graines, selon 3 classes : 0 graine, de 1 à 10 graines, plus de 10 graines.

\section{Mesures chimiques}

Les mesures physiques terminées, les tomates sont découpées en morceaux, mises en sac scellé, surgelées à $-40^{\circ} \mathrm{C}$, puis stockées en chambre froide à $-20^{\circ} \mathrm{C}$. Les fruits sont regroupés de façon à réduire à 2 le nombre d'échantillons à analyser par plante. Pour ce faire, chacun des lots est scindé en 2 parties: récolte 1 (bouquets 1 et 2) et récolte 2 (bouquets 3 et 4).

Dans le cas de "Apédice», la limite entre les 2 récoltes est fixée au 17 juin: en effet, jusqu'à cette date, la très grande majorité des fruits des 2 lots parthénocarpiques (avec ou sans vibration des inflorescences) ne contiennent pas de graines, alors qu'à partir de cette date, la plupart en contiennent. Le regroupement des fruits conduit à :

- lignée parthénocarpique, avec ou sans vibration des inflorescences : $1^{\text {re }}$ récolte : fruits sans graines $; 2^{\mathrm{e}}$ récolte : fruits avec plus de 10 graines;

- lignées non parthénocarpiques: $1^{\mathrm{rc}}$ et $2^{\mathrm{C}}$ récoltes : fruits avec plus de 10 graines.

Dans le cas de « Monalbo » et «Porphyre », les fruits de la lignée parthénocarpique ne contiennent pas de graines dans leur très grande majorité, et ceci quelle que soit la date de récolte des fruits, et que les inflorescences aient été vibrées ou non. De ce fait, la séparation entre les 2 récoltes est fixée au numéro d'ordre chronologique moyen de chaque lot, soit entre les 23 et 27 juin pour "Monalbo", les 21 et 25 juin pour "Porphyre ». Pour ces 2 types variétaux, la situation est donc la suivante:

- lignée parthénocarpique, avec ou sans vibration des inflorescences : $1^{\text {re }}$ et $2^{\mathrm{e}}$ récoltes : fruits sans graines ; - lignées non parthénocarpiques: $1^{\mathrm{re}}$ et $2^{\mathrm{C}}$ récoltes: fruits avec plus de 10 graines.

Les fruits d'une même plante satisfaisant ces conditions sont associés (jusqu'à 5 fruits au maximum) pour constituer un échantillon destiné aux analyses chimiques. Les fruits contenant de 1 à 10 graines ont été éliminés, de façon à avoir des fruits nettement parthénocarpiques ou nettement fécondés. Finalement 1741 fruits ont ainsi été retenus pour les analyses chimiques, et ont permis de constituer 377 échantillons sur un total théorique de 384 (2 échantillons pour chacune des 192 plantes cultivées). Des plantes manquantes, des accidents de manipulation, expliquent cette différence de 7 échantillons.

\section{Protocole des mesures chimiques}

Les morceaux des tomates regroupées (graines comprises quand il y en a) sont broyés en continu sous un léger flux d'azote liquide de telle sorte que la poudre issue du broyage ait une température de $-80^{\circ} \mathrm{C}$. Cette poudre est récupérée dans un sac plastique qui, une fois scellé, permet son entreposage à $-20^{\circ} \mathrm{C}$ jusqu'au moment de l'analyse.

Douze paramètres physico-chimiques sont étudiés pour chaque échantillon issu du regroupement des tomates. Parmi ceux-ci, l'acidité titrable, les acides organiques et les sucres réducteurs, peuvent être reliés à la qualité gustative; la matière sèche, les sucres réducteurs, l'indice de réfraction, aux aptitudes technologiques, en particulier pour les tomates de conserve. Les mesures de carotène et de vitamine $\mathrm{C}$ ont trait à des caractéristiques de qualité nutritionnelle.

Les méthodes d'analyse habituellement utilisées au laboratoire (BURET \& DUPRAT, 1983) sont appliquées.

\section{- Indice de réfraction, $p H$ et conductivité du jus}

L'indice de réfraction à $20^{\circ} \mathrm{C}\left({ }^{\circ}\right.$ Brix) et le $\mathrm{pH}$ sont mesurés directement sur le jus obtenu après filtration sur étamine (ouverture de maille : 50 meshes) d'environ $10 \mathrm{~g}$ de poudre fraîche décongelée.

La conductivité est déterminée à $25^{\circ} \mathrm{C}$ sur le jus dilué au $1 / 10^{\mathrm{e}}$ par de l'eau bidistillée bouillie. Les résultats sont exprimés en milliSiemens $(\mathrm{mS})$.

\section{- Acidité titrable, acide citrique, acide malique, rapport citrique/malique, sucres réducteurs}

Un échantillon d'environ $10 \mathrm{~g}$ de poudre fraîche congelée, pesé exactement, est décongelé en présence d'environ 3 fois son poids, exactement déterminé, d'eau bidistillée bouillie puis filtré sur étamine.

L'acidité titrable est déterminée par titrimétrie en neutralisant $2 \mathrm{ml}$ du filtrat par la soude $\mathrm{N} / 200$ jusqu'à pH 8,1 (méthode AOAC 22059). La teneur en acide est exprimée en méq $\mathrm{H}^{+}$pour $100 \mathrm{~g}$ de matière fraîche. 
Les acides malique et citrique sont dosés dans le filtrat dilué au $1 / 20^{\mathrm{c}}$, par voie enzymatique suivant respectivement les méthodes de Poux (1969) et de MoEllering \& Gruber (1966). Les résultats sont exprimés en méq $\mathrm{H}^{+}$ pour $100 \mathrm{~g}$ de matière fraîche.

Le rapport citrique/malique est calculé.

Les sucres réducteurs sont dosés dans le filtrat dilué de moitié, selon la méthode colorimétrique automatisée décrite par BITTNER \& MANNING (1967), basée sur la formation d'un complexe coloré avec la néocuproïne. Les résultats sont exprimés en $\mathrm{g}$ d'équivalent glucose pour $100 \mathrm{~g}$ de matière fraîche.

\section{- Teneur en matière sèche}

Un échantillon d'environ $10 \mathrm{~g}$ de poudre congelée, pesé exactement, est placé dans un sac de polyéthylène, préalablement taré. Après cryodéshydratation, le sac est soudé rapidement puis pesé. Les résultats sont exprimés en $\mathrm{g}$ de matière sèche pour $100 \mathrm{~g}$ de matière fraîche.

\section{- Teneur en vitamine $C$}

Le dosage de la vitamine $\mathrm{C}$ est effectué sur une prise d'environ $5 \mathrm{~g}$ de poudre congelée, pesée exactement, selon la méthode fluorimétrique automatique décrite par EGBERG et al. (1977). Les résultats sont exprimés en $\mathrm{mg}$ pour $100 \mathrm{~g}$ de matière fraîche.

\section{- Teneurs en lycopène et carotène}

Les pigments sont extraits successivement à l'acétone et à l'éther de pétrole. Les dosages sont réalisés selon la méthode de Lime \& GrifFITHS (1957), par spectroscopie d'absorption à 2 longueurs d'onde (451 et $503 \mathrm{~nm}$ ). Les résultats sont exprimés en microgrammes pour $100 \mathrm{~g}$ de matière fraîche.

A l'intérieur de chaque type variétal et pour chaque caractère mesuré, les moyennes de chacun des 4 lots sont comparées au moyen du test $\mathrm{t}$ de Student.

\section{RÉSULTATS}

\section{A. Résultats agronomiques}

Ils sont rassemblés dans le tableau 1.

\section{Comparaison des 2 lignées témoins non parthénocar- piques}

On ne note pas de différences significatives entre ces 2 lignées, pour chacun des 3 types variétaux (à l'exception du rendement précoce le 21 juin chez « Monalbo »). On peut donc estimer que le nombre de rétrocroise-

TABLEAU

Résultats agronomiques: hauteur des plantes, rendements, facteurs du rendement. Agronomic results : plant height, yield, yield components.

\begin{tabular}{|c|c|c|c|c|c|c|c|c|c|c|c|c|c|}
\hline \multirow{2}{*}{$\begin{array}{l}\text { Type } \\
\text { variétal }\end{array}$} & \multirow{2}{*}{ Génotype } & \multirow{2}{*}{$\begin{array}{c}\text { Traitement } \\
\text { inflorescences }\end{array}$} & \multirow{2}{*}{$\begin{array}{l}\text { Hauteur plantes } \\
\text { au } 6^{\mathrm{e}} \text { bouquet } \\
(\mathrm{cm})\end{array}$} & \multicolumn{8}{|c|}{ Rendements cumulés ( $\mathrm{kg} /$ plante) à différentes dates } & \multirow{2}{*}{$\begin{array}{l}\text { Nombre } \\
\text { fruits } \\
\text { par plante }\end{array}$} & \multirow{2}{*}{$\begin{array}{r}\text { Poids } \\
\text { moyen } \\
\text { fruit }(\mathrm{g})\end{array}$} \\
\hline & & & & $31-5$ & $7-6$ & $14-6$ & $21-6$ & $28-6$ & $6-7$ & $19-7$ & $30-7$ & & \\
\hline \multirow[t]{6}{*}{ Apédice } & pat-2/pat-2 & non vibré & $103 \mathrm{~b}$ & $0,40 \mathrm{a}$ & $0,60 \mathrm{a}$ & 0,79 & 1,17 & 1,62 & 2,09 & $2,63 \mathrm{c}$ & - & 47,7 & $55,1 \mathrm{~b}$ \\
\hline & pat-2/pat-2 & vibré & $106 \mathrm{~b}$ & $0,44 \mathrm{a}$ & 0,64 a & 0,83 & 1,30 & 1,79 & 2,22 & $2,78 \mathrm{bc}$ & - & 46,0 & $60,3 \mathrm{~b}$ \\
\hline & $+1+$ sœur & vibré & $114 \mathrm{a}$ & $0,16 \mathrm{~b}$ & $0,31 \mathrm{~b}$ & 0,55 & 1,23 & 1,86 & 2,23 & $3,12 \mathrm{ab}$ & - & 45,1 & 69,7 a \\
\hline & $+1+$ récurrent & vibré & $113 \mathrm{a}$ & $0,22 \mathrm{~b}$ & $0,39 \mathrm{~b}$ & 0,66 & 1,27 & 1,90 & 2,34 & $3,21 \mathrm{a}$ & - & 45,1 & $71,2 \mathrm{a}$ \\
\hline & \multicolumn{2}{|c|}{ ppds 0,05} & - & 0,12 & 0,15 & NS & NS & NS & NS & 0,35 & - & NS & 6,7 \\
\hline & \multicolumn{2}{|c|}{ Coefficient variation } & - & 22 & 17 & 19 & 12 & 9 & 10 & 7 & - & 7 & 6 \\
\hline \multirow[t]{6}{*}{ Monalbo } & pat-2/pat-2 & non vibré & $88 \mathrm{~b}$ & $\ldots$ & - & $-\cdots$ & $0,94 \mathrm{a}$ & 1,63 & 1,93 & $2,66 \mathrm{bc}$ & 3,32 & $50,7 \mathrm{ab}$ & $65,7 \mathrm{ab}$ \\
\hline & pat $-2 /$ pat -2 & vibré & $87 \mathrm{~b}$ & $\ldots$ & & - & $0,73 \mathrm{bc}$ & 1,44 & 1,81 & $2,51 \mathrm{c}$ & 3,17 & $51,7 \mathrm{a}$ & $61,0 \mathrm{~b}$ \\
\hline & $+/+$ sceur & vibrć & $95 \mathrm{a}$ & - & - & - & $0,79 \mathrm{~b}$ & 1,54 & 2,18 & $3,22 \mathrm{a}$ & 3,60 & 48,4 bc & $74,4 \mathrm{a}$ \\
\hline & $+/+$ récurrent & vibré & $88 \mathrm{~b}$ & - & - - & - & $0,66 \mathrm{c}$ & 1,34 & 2,09 & $3,20 \mathrm{ab}$ & 3,59 & $46,4 \mathrm{c}$ & 75,6 a \\
\hline & \multicolumn{2}{|c|}{ ppds 0,05} & - & - & - & - & 0,10 & NS & NS & 0,56 & $\mathrm{NS}$ & 2,7 & 11,7 \\
\hline & \multicolumn{2}{|c|}{ Coefficient variation } & & - & - & - & 7 & 11 & 11 & 11 & 12 & 3 & 10 \\
\hline \multirow[t]{6}{*}{ Porphyre } & pat-2/pat-2 & non vibré & - & - & - & 0,22 & $1,60 \mathrm{a}$ & 2,37 & 2,62 & 3,21 & - & 22,1 & $145,2 \mathrm{a}$ \\
\hline & pat $-2 /$ pat -2 & vibré & - & - & - & 0,23 & $1,67 \mathrm{a}$ & 2,17 & 2,35 & 3,10 & - & 23,0 & $134,2 \mathrm{~b}$ \\
\hline & $+1+$ sceur & vibré & - & - & - & 0,21 & $1,17 \mathrm{~b}$ & 2.20 & 2,77 & 3,19 & - & 22,3 & $143,7 \mathrm{ab}$ \\
\hline & $+/+$ récurrent & vibré & - & - & - & 0,17 & $1,21 \mathrm{~b}$ & 2,13 & 2,86 & 3,47 & - & 23,2 & $149,9 \mathrm{a}$ \\
\hline & \multicolumn{2}{|c|}{ ppds 0,05} & - & - & - & NS & 0,35 & NS & NS & NS & - & NS & 9,9 \\
\hline & \multicolumn{2}{|c|}{ Coefficient variation } & $\ldots .$. & - & - & 52 & 14 & 8 & 8 & 10 & -- & 11 & 4 \\
\hline
\end{tabular}

Des lettres différentes indiquent une différence significative au seuil 0,05 (comparaison à l'intérieur d'un même type variétal).

Hauteur des plantes: comparaison de moyennes avec le test $t$ de Student (les plantes de Porphyre, à croissance déterminée, n'ont pas été mesurées)

Rendements et facteurs du rendement : analyse en carré latin. 
ments réalisés a été suffisant pour retrouver les caractéristiques agronomiques des lignées utilisées cornme parents récurrents.

\section{Comparaison des 2 traitements, avec ou sans vibration} des inflorescences, chez la lignée parthénocarpique

Pour chacun des 3 types variétaux, on ne note pas de différences significatives entre les 2 traitements, à l'exception d'un rendement précoce plus élevé le 21 juin chez «Monalbo » non vibré, et d'un fruit plus petit chez «Porphyre» vibré. Rappelons que le traitement avec vibration, contrairement à ce que nous attendions, n'a augmenté que dans une très faible mesure la proportion de fruits avec graines (tabl. 2). Il n'est donc pas surprenant qu'il n'y ait pas de différences significatives entre les 2 traitements.

3. Comparaison des 2 lignées non parthénocarpiques d'une part, et de la lignée parthénocarpique d'autre. part

\section{a) Vigueur et hauteur des plantes}

La hauteur des plantes est diminuée chez la version parthénocarpique de «Apédice ». Cette diminution de la longueur des entre-nœuds, associée à une forte diminution de la vigueur, a déjà été observée chez cette lignée dans d'autres conditions de culture (Philouze, 1984). Le gène pat-2 semble bien responsable du manque de vigueur dans le type «Apédice»; la lignée témoin sœur de la lignée parthénocarpique a en effet une vigueur tout à fait normale.

Chez «Monalbo », les plantes de la lignée parthénocarpique sont en moyenne de même taille que celles du parent récurrent. Cependant, on est en présence d'une hétérogénéité dans la hauteur des plantes du matériel parthénocarpique (variance élevée), qui se traduit par la présence de quelques plantes nettement plus chétives que les autres. Cette observation souligne une sensibilité du matériel parthénocarpique aux conditions de culture et de milieu. Les techniques culturales appliquées dans cet essai sont appropriées aux cultivars normaux, alors que le matériel parthénocarpique demanderait la mise au point de techniques spécifiques.

\section{b) Précocité}

D'une façon générale, le matériel parthénocarpique se montre plus précoce que les lignées témoins correspondantes. Il y a des différences significatives entre versions parthénocarpiques et versions normales de "Apédice») pour les rendements des 31 mai et 7 juin, chez « Monalbo » non vibré le 21 juin, chez «Porphyre » le 21 juin.

\section{c) Rendement total}

Le rendement total est significativement plus faible chez "Apédice» parthénocarpique que chez les témoins; plus faible, mais de façon non significative, chez «Monalbo» parthénocarpique et chez «Porphyre » parthénocarpique.

\section{d) Facteurs du rendement}

Cette différence dans le rendement total ne résulte pas d'une différence dans le nombre de fruits (qui est au contraire plus élevé chez «Monalbo » parthénocarpique), mais d'une diminution de la taille du fruit : elle est nette chez «Apédice» et «Monalbo», et chez «Porphyre » vibré; par contre les fruits récoltés sur des inflorescences non vibrées de «Porphyre » parthénocarpique sont de même grosseur que les fruits de lignées témoins.

Les résultats agronomiques de cet essai conduit sous serre plastique très peu chauffée sont tout à fait comparables à ceux obtenus avec le même matériel (obtenu après 4 rétrocroisements seulement) sous serre verre chauffée en culture de printemps (PHILouzE, 1984). Nous retrouvons la très faible vigueur et le faible rendement de "Apédice » parthénocarpique, la diminution de la taille du fruit surtout chez les versions parthénocarpiques de «Apédice » et « Monalbo », et à un degré moindre chez «Porphyre» (comme dans l'essai présent, si le traitement avec vibration des inflorescences éntraîne chez ce dernier type variétal une diminution du poids des fruits, ce n'est pas le cas avec le traitement sans vibration des inflorescences).

\section{B. Caractéristiques physico-chimiques des fruits}

1. Caractéristiques physiques (tabl. 2)

a) Présence/absence de graines

Quel que soit le type variétal, la très grande majorité des fruits des lignées normales vibrées contiennent des graines. Chez les lignées parthénocarpiques, la majorité des fruits ne renferment pas de graines. La vibration des inflorescences augmente la proportion de fruits avec graines, mais dans des limites relativement réduites, nettement plus réduites que dans un essai conduit sous serre verre chauffée (PHILouze, 1984). Chez "Apédice " parthénocarpique, environ la moitié des fruits ne renferment pas de graines. Chez «Monalbo » et «Porphyre », la majorité des fruits des versions parthénocarpiques ne renferment pas de graines, même après vibration des inflorescences, et ce jusqu'aux dernières récoltes.

b) Mesures subjectives: notations de 3 caractéristiques internes des fruits

\section{- Importance des fruits creux}

Par rapport aux témoins, la version parthénocarpique de « Apédice » a moins de fruits creux, celle de « Monalbo » en a plus, celle de «Porphyre » en a autant. 
TABLEAU 2

Caracteristiques phlsiques des fruits.

Physical characteristics of the fruits.

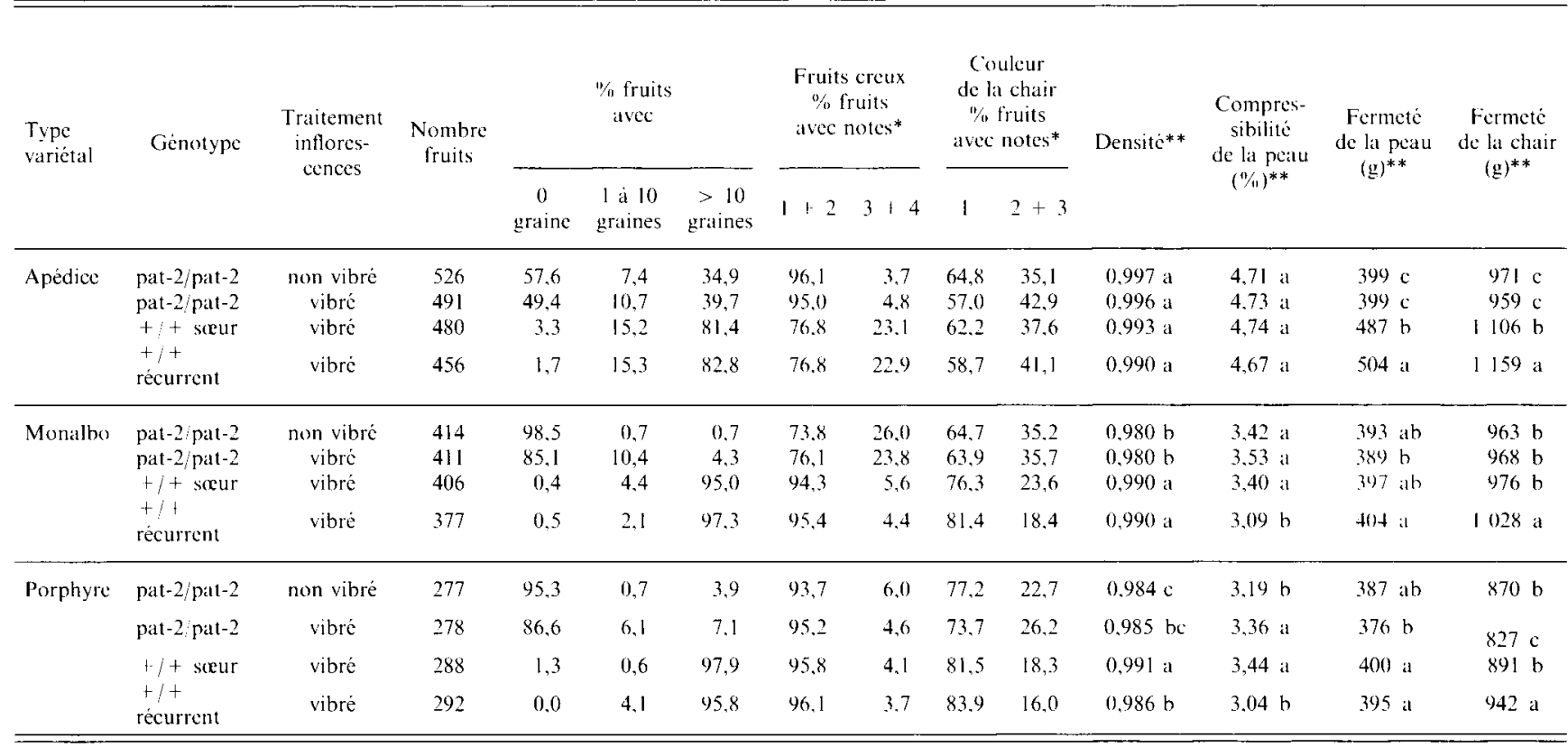

* Fruits creux : notes de I (fruit plein) à 4 (fruit très creux).

Couleur de la chair : note $1=$ orange-rouge, $2=$ rouge, 3 -: rouge foncé.

** Des lettres différentes indiquent une différence significative au seuil 0,05 (comparaison à l'intéricur d'un mème type variétal, avec le test $t$ do Student).

- Couleur du gel (résultats non présentés) et couleur de la chair

Les versions parthénocarpiques de « Monalbo » et de "Porphyre" ont tendance à avoir un gel un peu plus vert que les versions normales: en moyenne $15 \mathrm{p} .100$ des fruits de "Monalbo" parthénocarpique ont la note 1 (couleur vert-jaune du gel) contre 2 p. 100 des fruits des lignées témoins; chez "Porphyre», ces chiffres sont respectivement de 8 p. 100 et 1 p. 100 ; parallèlement, la chair présente une coloration rouge plus marquée. En revanche, aucune différence ne peut être notée entre les différentes versions de «Apédice».

\section{c) Mesures objectives}

\section{- Densité}

Les fruits des lignées parthénocarpiques de « Monalbo " et " Porphyre " sont moins denses que les fruits des lignées normales; on ne note pas de différence chez "Apédice».

\section{- Compressibilité de la peau, fermeté de la péau et} fermeté de la chair

Pour les trois variétés, la compressibilité de la peau est toujours la plus faible pour la lignée récurrente. Toutefois chez "Apédice», les différences ne sont pas significatives par rapport aux 3 autres lignées.

Les fermetés de la peau et de la chair sont toujours inférieures chez le matériel parthénocarpique. Les différences avec les lignées témoins sont significatives chez
"Apédice». Remarquons que la fermeté des lignées témoins, sœurs des lignées parthénocarpiques, est plus faible que celle des lignées récurrentes; un nombre de rétrocroisements insuffisant peut être incriminé dans ce cas.

Pour résumer ce chapitre sur les caractéristiques physiques des fruits, disons que les fruits des versions parthénocarpiques sont sensiblement moins fermes que les témoins; chez « Monalbo » et "Porphyre », la chair est plus rouge, le gel plus vert. Ces différences de coloration ne semblent pas résulter d'un biais de récolte (degré de maturité des fruits). Elles pourraient avoir comme origine des différences de comportement physiologique du gel et de la pulpe, notamment des vitesses de dégradation et de synthèse des pigments.

Dans le cas de "Apédice", on trouve aux alentours du 17 juin un mélange de fruits avec et sans graines chez la lignée parthénocarpique. Il a donc été possible, sur une période d'une dizaine de jours autour de cette date, de disposer de suffisamment de fruits dans chaque catégorie (à l'exception de la lignée normale sœur de la lignée parthénocarpique) pour comparer ces caractéristiques. Les résultats sont donnés dans le tableau 3. On trouve ici, alors que cela ne ressortait pas sur l'ensemble des fruits récoltés, un gel plus rouge chez les fruits sans graines, contrairement à ce qui se passe chez « Monalbo » et « Porphyre ». Par contre, la chair des fruits sans graines a tendance à être plus rouge, comme chez "Monalbo » et «Porphyre ». Les fruits sans graines de la lignée parthénocarpique sont significativement moins lourds que les fruits avec graines de la même lignée, eux-mèmes significativement moins lourds que les fruits de la lignée récurrente. On ne peut tirer aucune conclusion pour la densité et la compressibilité de la peau. Les 
TABLEAU 3

Caractéristiques physiques de finits de Apédice récoltés anx alentours du 17 juin.

Physical characteristics of Apédice fruits harvested around June 17.

\begin{tabular}{|c|c|c|c|c|c|c|c|c|c|c|c|c|c|c|}
\hline \multirow[t]{2}{*}{ Génotype } & \multirow[t]{2}{*}{$\begin{array}{c}\text { Traitement } \\
\text { inflores- } \\
\text { cences }\end{array}$} & \multirow[t]{2}{*}{$\begin{array}{l}\text { Nombre } \\
\text { graines } \\
\text { par fruit }\end{array}$} & \multirow[t]{2}{*}{$\begin{array}{l}\text { Nombre } \\
\text { fruits }\end{array}$} & \multicolumn{2}{|c|}{$\begin{array}{l}\text { Fruits } \\
\text { creux } \\
\% \text { fruits } \\
\text { avec notes* }\end{array}$} & \multicolumn{2}{|c|}{$\begin{array}{c}\text { Coulcur } \\
\text { du gel } \\
\% \text { fruits } \\
\text { avec notes* }\end{array}$} & \multicolumn{2}{|c|}{$\begin{array}{l}\text { Coulcur de } \\
\text { la chair } \\
\% \text { fruits } \\
\text { avec notes* }\end{array}$} & \multirow[t]{2}{*}{$\begin{array}{c}\text { Poids } \\
\text { fruits }(\mathrm{g})\end{array}$} & \multirow[t]{2}{*}{ Densité } & \multirow[t]{2}{*}{$\begin{array}{l}\text { Compres- } \\
\text { sibilité de } \\
\text { la peau } \\
(0 / 0)\end{array}$} & \multirow[t]{2}{*}{$\begin{array}{c}\text { Fermeté } \\
\text { de la peau } \\
\text { (g) }\end{array}$} & \multirow[t]{2}{*}{$\begin{array}{l}\text { Fermeti } \\
\text { de la chair } \\
\text { (g) }\end{array}$} \\
\hline & & & & $1+2$ & $3+4$ & $1+2$ & $3+4$ & 1 & $2+3$ & & & & & \\
\hline \multirow[t]{2}{*}{ pat-2 patt-2 } & non vibré & $\begin{array}{c}0 \\
>10\end{array}$ & $\begin{array}{l}80 \\
35\end{array}$ & $\begin{array}{c}96.3 \\
100\end{array}$ & $\begin{array}{l}3.7 \\
0\end{array}$ & $\begin{array}{l}17.5 \\
80\end{array}$ & $\begin{array}{l}82.5 \\
20\end{array}$ & $\begin{array}{l}33,8 \\
82,9\end{array}$ & $\begin{array}{l}66.2 \\
17,1\end{array}$ & $\begin{array}{ll}46.1 & c \\
59.9 & b\end{array}$ & $\begin{array}{l}1,000 \text { al } \\
1,001\end{array}$ & $\begin{array}{l}5,48 \text { il } \\
4,91 \quad \text { b }\end{array}$ & $\begin{array}{ll}372 & c \\
410 & b\end{array}$ & $\begin{array}{ll}868 & b \\
929 & b\end{array}$ \\
\hline & vibré & $\begin{array}{c}0 \\
>10\end{array}$ & $\begin{array}{l}62 \\
54\end{array}$ & $\begin{array}{l}91.9 \\
98,1\end{array}$ & $\begin{array}{l}8.1 \\
1,9\end{array}$ & $\begin{array}{l}14.5 \\
38,9\end{array}$ & $\begin{array}{l}85.5 \\
61.1\end{array}$ & $\begin{array}{l}21,0 \\
42,6\end{array}$ & $\begin{array}{l}79.0 \\
57,4\end{array}$ & $\begin{array}{l}45.4 c \\
60.9 \mathrm{~b}\end{array}$ & $\begin{array}{l}0,996 \mathrm{~b} \\
1.002 \mathrm{a}\end{array}$ & $\begin{array}{ll}5,00 & b c \\
5,36 & a b\end{array}$ & $\begin{array}{l}354 c \\
433 \mathrm{~b}\end{array}$ & $\begin{array}{ll}870 & b \\
932 & b\end{array}$ \\
\hline$t+$ récurrent & vibré & $>10$ & 62 & 80.6 & 19.4 & 50 & 50 & 61,3 & 38.7 & $7[.9$ a & $0,994 \mathrm{~b}$ & $4,72 \mathrm{c}$ & $494 \mathrm{a}$ & 1161 al \\
\hline
\end{tabular}

Des lettres diférentes indiquent une différence significative cu scuil 0,05 (comparaisons à Yintérieur d’un même type variétal, avec le test 1 de Student).

* Fruits creux : notes de 1 (fruit plein) à 4 (fruit très creux).

Couleur du gel : note 1 : vert-jaune, 2 : orangé-rouge, $3:$ rouge, 4 : rouge foncé.

Couleur de la chair: note 1 : orangé-rouge, 2 : rouge, 3 : rouge foncé.

fruits sans graines de la lignée parthénocarpique ont des fermetés moindres pour la peau et la chair, que les fruits avec graines de la même lignée, eux-mêmes significativement moins fermes que les fruits du parent récurrent.

\section{Caractéristiques chimiques}

Douze données sont présentées dans les tableaux 4 et 5 .

\section{a) Acidité titrable, teneur en acide citrique, teneur en acide malique}

Les fruits du matériel parthénocarpique sont généralement moins acides que les fruits du matériel normal. Cette remarque se confirme pour l'acide citrique (différences le plus souvent significatives) et de manière moins nette pour l'acide malique où l'on note quelques différences mais pas de tendance générale. L'effet de la parthénocarpie sur l'acidité titrable est quantitativement plus marqué chez " Porphyre». Pour la teneur en acide citrique, les différences par rapport au matériel normal sont les plus importantes chez «Porphyre » et "Monalbo". Par contre, l'influence sur la teneur en acide malique est variable. Ainsi, à la première récolte, l'acidité malique de "Apédice» parthénocarpique est plus faible que celle des témoins ; c'est le contraire chez "Monalbo ". Notons par ailleurs la très faible teneur en acide malique du type «Porphyre».

\section{b) Rapport citrique/malique et $\mathrm{pH}$}

Chez les parthénocarpiques, le rapport citrique/malique est plus faible que chez les témoins, à l'exception de la première récolte chez "Apédice». En ce qui concerne le $\mathrm{pH}$, les différences observées ne permettent pas de dégager de tendance générale : elles sont du même ordre de grandeur que pour d'autres variétés étudiées (BURFT \& DUPRAT, 1983).

\section{c) Conductivité du jus}

En général, la conductivité du matériel parthénocarpique est inférieure à celle des témoins.

\section{d) Indice de réfraction, teneur en sucres réducteurs et teneur en matière sèche}

Les différences observées pour l'indice de réfraction ne permettent pas de dégager de tendance générale, sauf peut-être chez "Porphyre » où les fruits parthénocarpiques présentent un indice de réfraction plus faible que les témoins. Quand il y a des différences de teneur en matière sèche, elles vont dans le sens : teneur chez les parthénocarpiques inférieure à celle des témoins. C'est le cas en particulier pour la $2^{\mathrm{e}}$ récolte de "Monalbo » et pour les 2 récoltes de "Porphyre ». En revanche, pour les sucres réducteurs les teneurs sont comparables.

\section{e) Teneur en lycopène}

Chez «Monalbo » et chez «Porphyre », la teneur en lycopène des parthénocarpiques est significativement plus élevée que celle des témoins. Ce n'est pas le cas chez "Apédice ». Ces résultats sont à mettre en relation avec les notations sur la couleur de la chair et ne doivent pas être attribuées à des différences de maturité lors de la récolte mais plutôt à une caractéristique propre aux fruits parthénocarpiques.

\section{f) Teneur en carotène (provitamine A) et teneur en vitamine $C$}

Rien de net ne se dégage pour la teneur en carotène, sauf chez « Monalbo » où la teneur des fruits parthénocarpiques est plus élevée que celle des témoins. Dans tous les cas, la teneur en vitamine $\mathrm{C}$ des lignées parthénocarpiques est inférieure à celle des fruits de leurs lignées sœurs non parthénocarpiques. La position des 


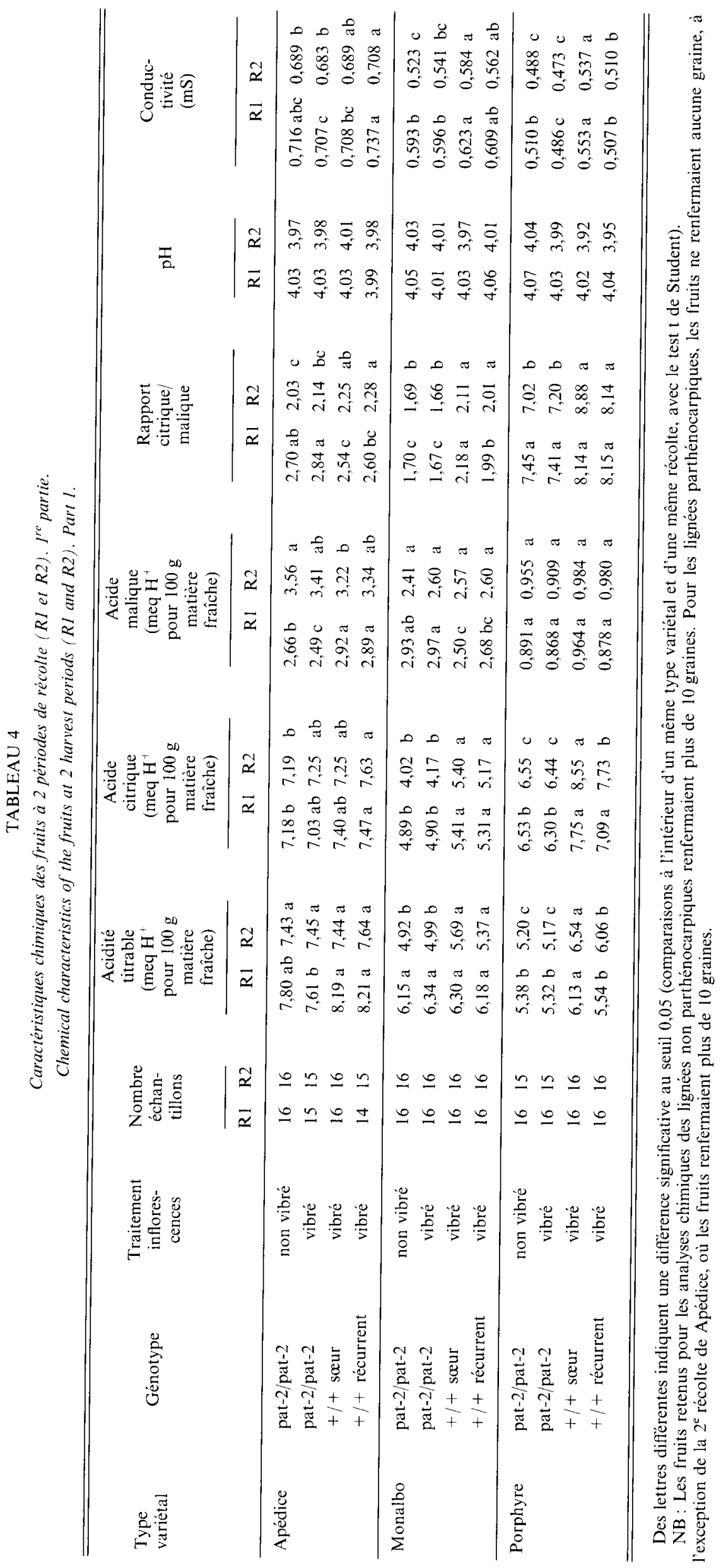




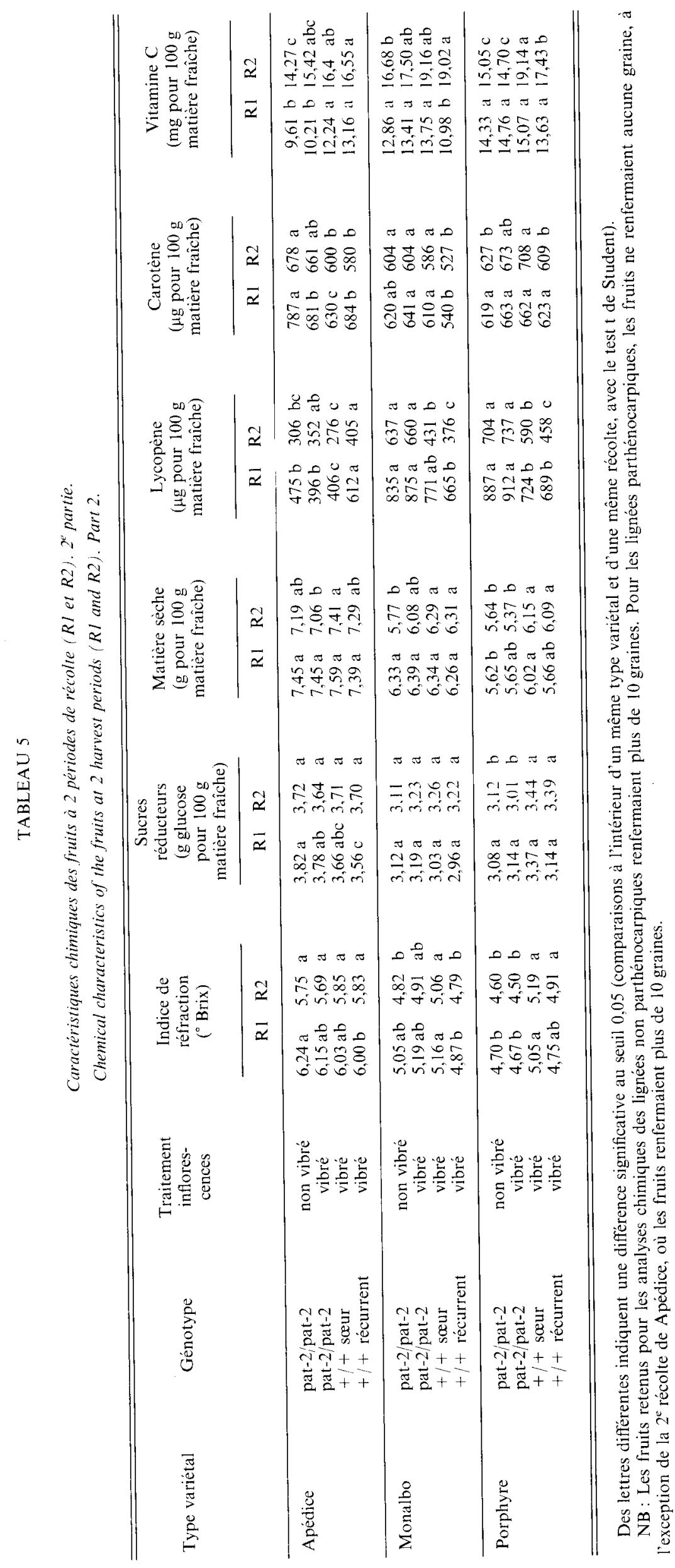


lignées récurrentes est variable, selon le type variétal et selon la récolte. Par ailleurs, la teneur des fruits de la $2^{\mathrm{e}}$ récolte est toujours supérieure, et de façon sensible, à celle des fruits de la $I^{\text {re }}$ récolte. Faut-il attribuer la teneur moindre des lignées parthénocarpiques à un effet du gène pat-2 lui-même, ou au fait que le matériel parthénocarpique est plus précoce?

\section{DISCUSSION ET CONCLUSION}

Comme nous l'avons déjà souligné (PhILouze, 1984), aucune caractéristique agronomique ne semble modifiée de façon systématique par le gène pat-2, si ce n'est la présence de nombreux fruits sans graines, et une amélioration de la précocité. Cependant, le gène pat-2 entraîne un manque de vigueur, au moins chez «Apédice». Chez "Monalbo », on note la présence de quelques plantes particulièrement chétives dans le matériel parthénocarpique, alors que les plantes voisines sont tout à fait comparables aux plantes témoins. Ce premier résultat permet déjà deux observations: le sélectionneur devra tenir compte de l'effet du gène pat-2 sur la vigueur, et veiller à utiliser des géniteurs suffisamment vigoureux. D'autre part des techniques culturales appropriées au matériel parthénocarpique doivent à lévidence être mises au point. En effet, du fait d'une mise à fruit précoce sur des plantes peu vigoureuses, le rendement des bouquets supérieurs (nombre et poids des fruits) est très certainement inférieur à ce qu'on pourrait en attendre si on adoptait des techniques culturales appropriées, empêchant l'épuisement prématuré de la plante. Les fruits sans graines des lignées parthénocarpiques sont plus petits que ceux avec graines des lignées normales chez «Apédice » et «Monalbo »; la réponse est moins nette chez «Porphyre». Rappelons que chez « Severianin ", il est possible d'avoir des fruits sans graines de même poids que les fruits avec graines (PHILouze, 1985). Fort de ce résultat, le sélectionneur cherchera à utiliser un matériel de départ à fruits assez gros pour que, en cas de diminution de la taille des fruits, celle-ci reste malgré tout suffisante.

Les fruits sans graines de "Apédice" sont moins fermes (peau et chair) que les fruits avec graines, à l'intérieur de la même lignée parthénocarpique (tabl. 3). Cette diminution de fermeté peut constituer un handicap au niveau de la commercialisation. Du fait de différences variétales considérables pour ce caractère, il doit être possible d'obtenir du matériel parthénocarpique à fruits sans graines présentant une fermeté satisfaisante.

Le même type de raisonnement tient pour les caractéristiques chimiques sur lesquelles le gène pat-2 semble avoir quelque effet. Les différences observées entre fruits sans graines des lignées parthénocarpiques et fruits avec graines des lignées témoins sont souvent moins importantes que les différences que l'on peut trouver entre types variétaux (acidité titrable, acide citrique, acide malique, rapport citrique/malique, conductivité, indice de refraction, matière sèche), ou entre périodes de récolte (vitamine C). La comparaison entre les 2 lignées témoins à l'intérieur d'un même type variétal ne montre pas de différences au niveau des résultats agronomiques. Il y a parfois des différences au niveau des mesures physiques ou chimiques des fruits, mais ces différences ne vont pas toujours dans le même sens.

Toutes les comparaisons que nous nous étions proposé d'effectuer n'ont pu être faites, vu le nombre très insuffisant de fruits avec graines chez les versions parthénocarpiques de «Monalbo » et «Porphyre»; les seules comparaisons complètes concernent les caractéristiques physiques chez «Apédice ». Nous ne pouvons donc pas confronter de façon précise nos résultats avec ceux de Casas-Diaz et al. (1987) qui ont comparé les fruits avec ou sans graines à l'intérieur d'une même lignée parthénocarpique. Dans nos essais, les fruits sans graines des lignées parthénocarpiques n'ont pas un indice de réfraction plus élevé que celui des fruits avec graines des lignées témoins, leur $\mathrm{pH}$ n'est pas plus faible. Contrairement à ceux de CASAs-DiAz et al. (1987), nos résultats, obtenus avec des variétés et dans des conditions bien différentes, ne plaident pas en faveur de l'utilisation de la parthénocarpie due au gène pat-2 pour améliorer la teneur en sucres solubles ou en matière sèche. Cependant il conviendrait de vérifier ces résultats dans d'autres conditions de culture avec un matériel végétal diversifié (en particulier des types variétaux destinés à l'industrie).

Les interactions entre caractères (par exemple entre calibre et caractéristiques physico-chimiques des fruits, ou entre précocité et teneur en vitamine C) mériteraient d'être étudiées plus avant. Par ailleurs une étude de l'aptitude à la mise en marché (stockage au froid, durée de survie entre la récolte et la commercialisation) des lignées parthénocarpiques devrait permettre de définir le stade optimal de maturité pour la récolte, afin d'assurer le maintien de la qualité tout au long de la chaîne de distribution.

Ces résultats devraient permettre au sélectionneur de travailler de manière plus efficace, en tenant compte, par le choix de géniteurs appropriés, des modifications de certaines caractéristiques agronomiques ou physicochimiques des fruits qu'entraine le gène pat-2, tout en essayant de tirer parti au maximum de ses avantages, c'est-à-dire une mise à fruits et une précocité améliorées en conditions difficiles de température. L'absence de graines peut par ailleurs être considérée comme un critère de qualité, tant pour le marché de frais que pour les produits de type « $4^{\mathrm{c}}$ gamme».

\section{Regule 17 décembre 1987.} Accepté le 26 juin 1988.

\section{REMERCIEMENTS}

Les auteurs remercient les personnels techniques de la Station d'Amélioration des Plantes Maraîchères et du Laboratoire de Methodes Physiques d'Etude, qui, tres nombreux, ont participé à ce travail. 


\section{RÉFÉRENC:ES BIBLIOGRAPHIQUES}

Bittner D., Manning J., 1987. Automated necouproin glucose method: critical factors and normal values. Aulomation in analytical chemistry Publ. Technicon, 1-6.

Buret M., Duprat F., 1983. Aspects méthodologiques de l'étude de la qualité de la tomate (synthèse de 4 annés d'expérimentation en scrre et plein champ). Rapport interne, $64 \mathrm{p}$.

Casas-Diaz A. V., Hewitt J. D., Lapushner D., 1987. Effects of parthenocarpy on fruit quality in tomato. J. Amer. Soc. Hort. Sci., 112 (4), 634-637

Duprat F., Buret M., Nicolas J., Nicolas M., 1987. A methodology of fruit analysis. Proceedings of the 4th European Conference on Food Chemistry. FECS event n 108 - Loen (Norway) 1987/06/01-04, vol. 2, 533-538.

Egberg D. C., Potter R. H., Heroff J. C., 1977. Semi-automated method for the fluorometric determination of total vitamin $\mathrm{C}$ in food products. J. Assoc. Off. Anal. Chem., 60, 126-131.

Lime B. J., Griffiths F. P., 1957. Spectrophotometric methods for determining pigmentation. $\beta$. carotene and lycopene in ruby red grape fruil. J. Agric. Food Chem., 5 (12), 541-544.

Moellering H., Gruber W., 1966. Determination of citrate with citrate lyase. Anal. Biochem., 17, 369-376.

Nicolas J., Buret M., Duprat F., Nicolas M., Rothan C., Moras P., 1986. Effects of different conditions of cold storage upon physicochemical changes of kiwi fruit. Acta Hortic., 194, 261-272.

Philouze J., 1983. Parthénocarpie naturelle chez la tomate. I. Revue bibliographique. Agronomie, 3 (7), 611-620.

Philouze J., 1984. Comparisons between nearly isogenic parthenocarpic and normal tomato lines or hybrids. A new era in tomato breeding. Eucarpia Tomato Working Group. Synopses 9th meeting, IVT Wageningen, (Netherlands), 1984/05/22-24, 22-26.

Philouze J., 1985. Parthénocarpie naturelle chez la tomate. II. Etude d'une collection variétale. Agronomie, 5 (1), 47-54.

Poux C., 1969. Dosage enzymatique de lacide L-malique. Ann. Technol. Agric., 18, 359-366. 